\title{
Quantum tunneling in gap discrete breathers
}

\author{
V. I. Dubinko \\ vdubinko@mail.ru \\ NSC Kharkov Institute of Physics and Technology, 1 Akademicheskaya St., 61108, Kharkov, Ukraine \\ In many-body nonlinear systems, a special kind of lattice vibrations, namely, discrete breathers (DBs) can be excited either \\ thermally or by external triggering, in which the amplitude of atomic oscillations greatly exceeds that of harmonic oscillations \\ (phonons). Coherency and persistence of large atomic oscillations in DBs may have drastic effect on quantum tunneling due \\ to correlation effects discovered by Schrödinger and Robertson in 1930 and applied to the tunneling problem by Dodonov et \\ al (1980) and Vysotskii et al (2010). In the present paper, it is argued that DBs present the most natural and efficient way to \\ produce correlation effects due to time-periodic modulation of the potential well (or the Coulomb barrier) width and hence to \\ act as breather 'nano-colliders' (BNC) triggering low energy nuclear reactions (LENR) in solids. In particular, due to the large \\ mass difference between $\mathrm{H} / \mathrm{D}$ and the metal atoms, there is a gap in phonon spectrum of metal-hydride/deuteride crystals, \\ in which so called 'gap DBs' arise in the H/D sub-lattice resulting in time-periodic modulation of spacing between adjacent \\ $\mathrm{H} / \mathrm{D}$ and metal atoms. Tunneling probability for deuterium fusion in 'gap DBs' is shown to increase drastically with increasing \\ number of oscillations resulting in the observed LENR rate under heavy water electrolysis.
}

Keywords: phonon gap, discrete breathers, correlation effects, low energy nuclear reactions, nuclear active sites

\section{Introduction}

The tunneling through the Coulomb potential barrier during the interaction of charged particles presents a major problem for the explanation of low energy nuclear reactions (LENR) observed in solids [1-4].

The tunneling coefficient (TC) is the Gamow factor, given by

$$
G \approx \exp \left\{-\frac{2}{\hbar} \int_{r_{1}}^{r_{2}} d r \sqrt{2 \mu(V(r)-E)}\right\}
$$

Where $2 \pi \hbar$ is the Planck constant, $\mathrm{E}$ is the nucleus $\mathrm{CM}$ energy, $\mu$ is the reduced mass, $r_{1}, r_{2}$ are the two classical turning points for the potential barrier, which for the D-D reaction are given simply by $\mu=m_{D} / 2, V(r)=e^{2} / r$. For two D's at near to room temperature one has $G \sim 10^{-2760}$, which shows the need for some special conditions arising in solids under typical LENR conditions that help to overcome the Coulomb potential.

Corrections to the cross section of the fusion due to the screening effect of atomic electrons result in the so-called «screening potential», which acts as an additional energy of collision at the center of mass [5,6]. However, even the maximum screening potentials found in $\mathrm{Pt}(675 \mathrm{eV}), \mathrm{PdO}$ $(600 \mathrm{eV})$ and $\mathrm{Pd}(310 \mathrm{eV})$ are far too weak to explain LENR observed at temperatures, which are bellow melting point of solids (in E-cat type installations) or boiling point of liquids (under heavy water electrolysis).

The most promising and universal mechanism of the stimulation of nuclear reactions running at a low energy is connected with the formation of coherent correlated states of interacting particle, which ensures the large probability of the nuclear reactions under conditions, where the ordinary tunneling coefficient is negligible. This mechanism is based on the generalized uncertainty relation (UR) by SchrödingerRobertson [7,8], which takes into account correlations between coordinate and momentum operators. Correlation effects have been applied to the tunneling problem by Dodonov et al [9] and by Vysotskii et al [10-13] who demonstrated a giant increase of sub-barrier transparency caused by increasing correlation coefficient at special highfrequency periodic action on quantum system.

In this paper, we argue that such an action can be most naturally and effectively realized due to time-periodic modulation of the width of potential wells for atoms oscillating near gap discrete breathers (DBs). DBs are spatially localized large-amplitude vibrational modes in lattices that exhibit strong anharmonicity [14-23]. Due to the crystal anharmonicity, the frequency of atomic oscillations increase or decrease with increasing amplitude so that the DB frequency lies outside the phonon frequency band, which explains the weak DB coupling with phonons and, consequently, their robustness at elevated temperatures. DBs can be of one-frequency or many-frequency type [23]. In the former case, all atoms vibrate with the same frequancy, while in the latter case, they vibrate with different but commensurate frequencies. DBs can be excited either thermally or by external driving, as was observed experimentally $[17,18]$ and modelled in various physical systems [19-26].

Presently the interest of researchers has shifted to the study of the catalytic impact of DBs on the reaction rates in solids and on the biological functions of biopolymers $[26,27]$. Excitation of DBs in solids have been shown to result in a drastic amplification of chemical [27-30] and nuclear 
[31] reaction rates in their vicinity. In the former case, the amplification mechanism is based on modification of the classical Kramers escape rate from a potential well due to a periodic modulation of the well depth (or the reaction barrier height), which is an archetype model for chemical reactions since 1940 [32].

In the latter case, so-called gap DBs, which can arise in diatomic crystals such as metal hydrides/deuterides, have been argued to be the LENR catalyzers due to time-periodic closing of adjacent H/D atoms, which should enhance their fusion probability [31]. The main problem with this mechanism was that unrealistically small separation between atoms $(\sim$ $0.01 \AA$ ) would have attained in order to increase TC up to the level required for a noticeable LENR rate at the best choice of parameters. However, this estimate did not take into account correlations between coordinate and momentum operators arising in a DB due to cooperative nature of its dynamics. These effects are analyzed in the present paper.

\section{Formation of correlated states in non-stationary potential well}

\subsection{Schrödinger-Robertson uncertainty relation}

The tunneling effect for nuclear particles is closely related to the uncertainty relation (UR), which have been generalized with account of correlation effects independently by Schrödinger [7] and Robertson [8], which can be written in the following form [9]

$$
\begin{gathered}
\sigma_{x} \sigma_{p} \geq \hbar_{e f}^{2} / 4, \hbar_{e f}=\frac{\hbar}{\sqrt{1-r^{2}}} \\
\sigma_{x}=\left\langle\left(x-\langle x\rangle^{2}\right)\right\rangle, \sigma_{x}=\left\langle\left(p-\langle p\rangle^{2}\right)\right\rangle, \\
\sigma_{x p}=\langle\hat{x} \hat{p}+\hat{p} \hat{x}\rangle / 2-\langle x\rangle\langle p\rangle \\
r=\frac{\sigma_{x p}}{\sqrt{\sigma_{x} \sigma_{p}}}
\end{gathered}
$$

where $r$ is the correlation coefficient between the coordinate, $x$, and momentum, p. At $r=0$ (non-correlated state) Eq.(2) is reduced to the well-known Heisenberg UR, while in a general case, a nonzero $r$ in the UR can be taken into account by the formal substitution $\hbar \rightarrow \hbar_{e f}$, which designates a shift of the border between the classical and quantum descriptions of the same object in the transition from non-correlated to correlated state [13].

The most impressive consequence of correlation effects is a dramatic increase of the tunneling probability for a very low barrier transparency (tunneling probability) in the initially uncorrelated state $G_{r=0}<<1[13]$ :

$$
G_{r \neq 0} \approx \exp \left\{-\frac{2}{\hbar_{e f}} \int_{r_{1}}^{r_{2}} d r \sqrt{2 \mu(V(r)-E)}\right\}=\left(G_{r=0}\right)^{\sqrt{1-r^{2}}}
$$

which is within an order of magnitude close to the result of the exact calculation of the potential barrier transparency using rigorous quantum-mechanical methods [13]. From Eq.(6), it follows that when a coherent correlated state (CCS) with $|r| \rightarrow 1$ is formed, the product of the variances of the particle coordinate and momentum increases indefinitely, and the barrier becomes 'transparent':

$$
G_{|r| \rightarrow 1} \rightarrow 1 \text { even if } E<<V_{\max },
$$

A CCS can be formed in various quantum systems, such as a particle in a non-stationary potential well. The most relevant case in relation to DBs is considered bellow.

\subsection{Formation of CCS in oscillating potential well}

A model system considered by Vysotskii et al [11-13] for evaluation of the correlation coefficient is a non-stationary harmonic oscillator for a particle with the mass $\mathrm{M}$, coordinate $\mathrm{x}(\mathrm{t})$ placed in a non-stationary parabolic potential well,

$$
V(x, t)=M(x(t))^{2}(\omega(t))^{2} / 2
$$

with the eigenfrequency $\omega(t)$ changing periodically, which was shown to result in an increase of $|r(t)|$. This regime can be provided, e.g. at a constant well depth $V_{\max }$ and the potential well width $L(t)$ that changes periodically resulting in a timeperiodic modulation of the eigenfrequency as follows:

$$
L(t)=L_{0}\left(1+g_{\Omega} \cos \Omega t\right), L_{0}=\sqrt{8 V_{\max } / M \omega_{0}^{2}},
$$

where $L_{0}$ and $\omega_{0}$ are the initial parameters of the well before the action of correlated forces, $g_{\Omega}$ and $\Omega$ are the modulation amplitude and frequency, respectively.

Fig. 1 shows that the probability density $|\psi(x, r)|^{2}$ for the particle localization in the time-periodic well is very narrow for uncorrelated state $r=0$ (solid black), while it spreads significantly into the sub-barrier region for strongly correlated state $r=0.98$ at the times of the maximal coordinate dispersion (dash green) [10].

From a detailed analysis [11-13] it follows that the process of formation of strongly correlated coherent state

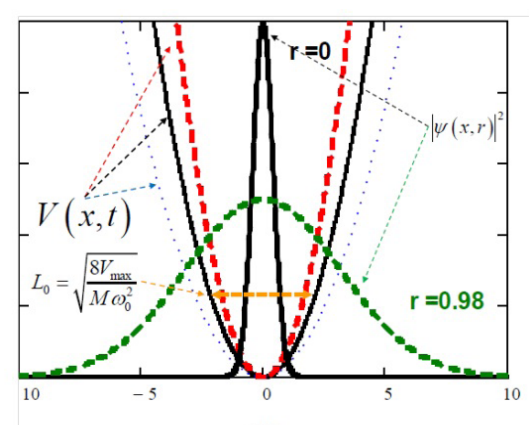

Fig. 1. Sketch of the time-periodic parabolic potential $V(x, t)$ at different moments of time corresponding to the initial (solid black), minimal (dash red) and maximal (dot blue) well width. Probability density $|\psi(x, r)|^{2}$ for the particle localization in the well and in the sub-barrier region is shown schematically for uncorrelated state $\mathrm{r}=0$ (solid black) and for strongly correlated state $\mathrm{r}=0.98$ at the times of the maximal coordinate dispersion (dash green) [10]. $x_{0}=\sqrt{\hbar / M \omega_{0}}$ is the half-width of the particle localization in the unperturbed well. 


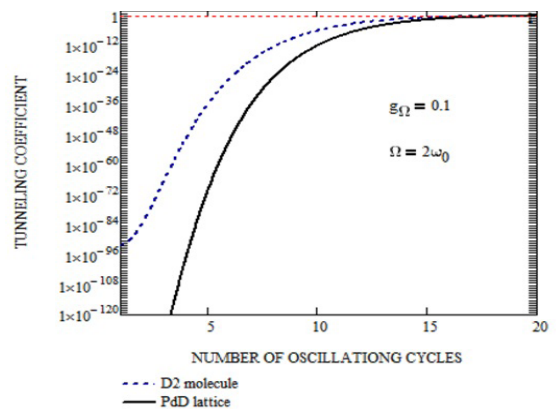

Fig. 2. Tunneling coefficient increase with increasing number of the well modulation cycles, $n=\omega_{0} t / 2 \pi$, evaluated by Eq.(11) for $\Omega=\omega_{0}$ (a); $\Omega \approx 2 \omega_{0}$ (b), $g_{\Omega}=0.1$ for two D-D equilibrium spacings: in a $\mathrm{D}_{2}$ molecule $\left(\mathrm{L}_{0}=0.74 \AA\right)$ and in the PdD crystal $\left(\mathrm{L}_{0}=a_{\mathrm{PdD}}(2 / 2)^{0.5} \approx 2.9 \AA\right)$. $a_{\mathrm{PdD}}=4.052 \AA$ is the PdD lattice constant at $295 \mathrm{~K}[33]$.

with $|r|_{\max } \rightarrow 1$ in response to the action of limited periodic modulation (Eq.9) is possible only at any of two conditions: (i) $\Omega=\omega_{0}$ (resonant formation) or (ii) $\Omega$ is close to $2 \omega_{0}$ (parametric formation): $\left|\Omega-2 \omega_{0}\right| \leq \mathrm{g}_{\Omega} \omega_{0}$. In these regimes, the correlation coefficient oscillates with time but its amplitude $|r| \max$ increases monotonously with the number of modulation cycles, $n=\omega_{0} t / 2 \pi$, resulting in a giant increase of the tunneling coefficient, as demonstrated in Fig.2, which shows the TC evaluated by Eq.(10) that takes into account both the electron screening [31] and the correlation effects [13]:

$$
G^{*}(L, r)=\exp \left\{-\frac{2 \pi e^{2}}{\hbar_{e f}(r)} \sqrt{\frac{\mu}{2\left(E+e^{2} / L\right)}}\right\},
$$

where $L$ is the minimum equilibrium spacing between $D$ atoms determined by electron screening, $E$ is their kinetic energy $\left(\sim \mathrm{eV} / 40\right.$ at room temperature) $<<$ screening energy $\sim e^{2} / L$. One can see that the difference in electron screening and the corresponding initial D-D distances in a D2 molecule $\left(L_{0}=0.74 \AA\right)$ and in the PdD crystal $\left(L_{0}=2.9 \AA\right)$ leads to a huge tunneling difference in the initial (uncorrelated) state, in which TC is negligible in both cases. However, with increasing number of modulation cycles, $h_{e f}(r)$ increases as well resulting in a giant increase of TC up to $\sim 1$ in several dozens of cycles for parametric formation $\Omega \approx 2 \omega_{0}$, which does not require exact coincidence of the frequencies [13].

The most important and nontrivial practical question now is how to realize such a periodic action at atomic scale? Modulation of the frequency of the optical phonon modes via excitation of the surface electron plasmons by a terahertz laser suggested in [13] as a driving force for the CCS formation is very questionable [31] (see also discussion in section 5), and it does not explain LENR observed in the absence of the laser driving. In the next section, we will consider a new mechanism based on the large-amplitude time-periodic oscillations of atoms naturally occurring in discrete breathers.

\section{Breather-induced time-periodic action on the potential landscape}

In order to develop a mechanism for DB-based LENR in diatomic crystals $\mathrm{PdD}$ and $\mathrm{NiH}$ we note that at ambient

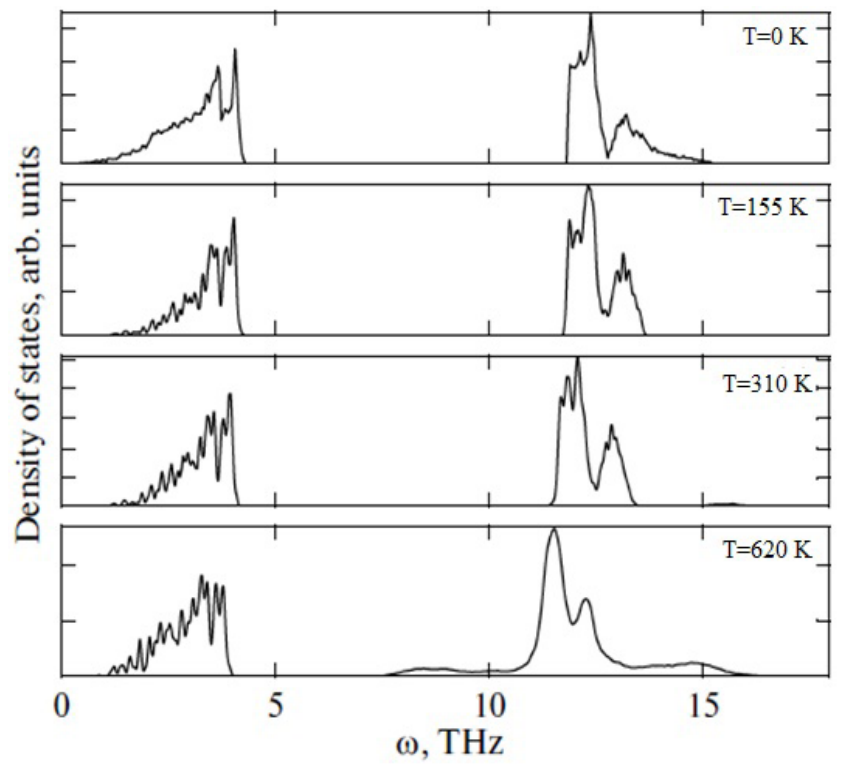

Fig. 3. DOS of the NaCl-type crystal for the weight ratio $m / M=0.1$. Reproduced from [20] Copyright by APS.

conditions, they crystallize in FCC structure with the space group of the $\mathrm{NaCl}$ structure [33-35]. Molecular dynamic (MD) simulations have revealed that diatomic crystals with Morse interatomic interactions typically demonstrate soft type of anharmonicity [19], which means that DB's frequency decreases with increasing amplitude, and one can expect to find so-called gap DBs with frequency within the phonon gap of the crystal. The large mass difference between $\mathrm{H}$ or D and the metal atoms is expected to provide a wide gap in phonon spectrum (Fig.3), in which DBs can be excited e.g. by thermal fluctuations at elevated temperatures as demonstrated by Kistanov and Dmitriev [20] for the different weight ratios and temperatures. Density of phonon states (DOS) of the NaCltype crystal for the weight ratio $m / M=0.1$ at temperatures ranging from $0 \mathrm{~K}$ to $620 \mathrm{~K}$ is shown in Fig.3a-d.

Fig.4 shows that DOS for $\mathrm{PdD}$ and $\mathrm{PdH}$ measured experimentally are qualitatively similar to DOS calculated for the NaCl-type crystals, while first-principles calculations [34] point out that phonon spectra in $\mathrm{PdD}$ and $\mathrm{PdH}$ are strongly

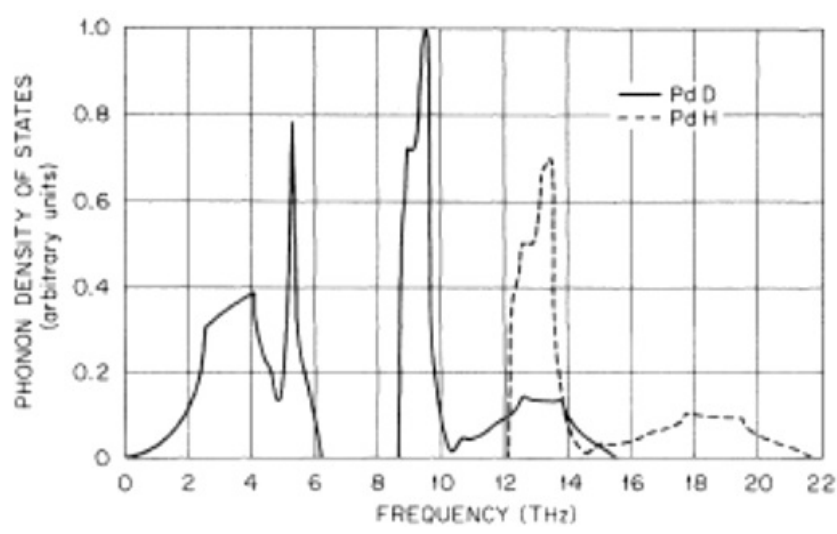

Fig. 4. DOS for PdD and PdH crystals based on the force constants obtained from the Born von Karman mode1 [33] fitted to the experimental results for $\mathrm{PdD}_{0.63}$ assuming that the forces in $\mathrm{PdD}$ and $\mathrm{PdD}_{0.63}$ were identical. Reproduced from [33] Copyright by APS. 


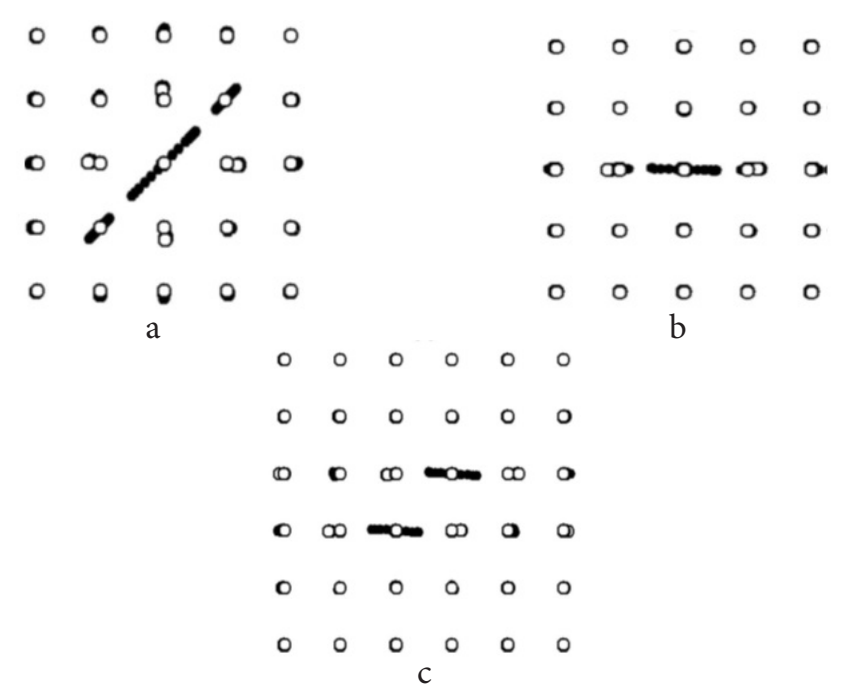

Fig. 5. Stroboscopic pictures showing motion of atoms for the DBs of three types: (a) $[110]_{1}$, (b) $[100]_{1}$, and (c) $[100]_{2}$, where figures in brackets describe polarization and the subscript indicates the number of the atoms oscillating with large amplitude. In panels (a) and (b) displacements of the atoms are multiplied by factor 7 , and in panel (c) by factor 5 . Heavy (light) atoms are shown by open (filled) circles. Reproduced from [19] Copyright by APS.

renormalized by anharmonicity.

The appearance of two additional broad peaks in the MD modelled DOS at elevated temperatures can be seen in Fig. 3d. The appearance of the peak in the gap of the phonon spectrum can be associated with the spontaneous excitation of gap DBs at sufficiently high temperatures, when nonlinear terms in the expansion of interatomic forces near the equilibrium atomic sites acquire a noticeable role. As the temperature increases, the lifetime and concentration of gap DBs in the light atom sub-lattice increase [21]. The appearance of the peak above the phonon spectrum at sufficiently high temperatures can be associated with the excitation of DBs of another type, which manifest the hard type nonlinearity. This conclusion agrees with a recent result by Zakharov et al [36] for another type of diatomic crystals, $\mathrm{Pt}_{3} \mathrm{Al}$, in which both soft type $\mathrm{DBs}$ and hard type DBs were modelled.

Dynamic structure of gap DBs has been revealed in [19] where they have been excited simply by shifting one light atom or two neighboring light atoms from their equilibrium positions while all other atoms were initially at their lattice positions and had zero initial velocities. In this way, for the weight ratio $m / M=0.1$, three types of stable DBs have been excited (Fig.5), frequencies of which are shown in Fig.6 as the functions of their amplitudes.

All three types of gap DBs are characterized by a high degree of spatial localization of atomic displacements with only one or two atoms having large amplitudes. They shift positions of neighboring atoms, which continue to oscillate with small (harmonic) amplitudes but with frequencies coinciding with DB frequencies shown in Fig.6. Degree of spatial localization at $m / M=0.1$ remains almost unchanged for about 5000 breather oscillations.

One can see that the maximum DB amplitude is about 0.34 $\AA$ for the polarization $[100]_{1}$, in which the nearest neighbor (heavy atom) is separated from the light atom by $3.125 \AA$. The

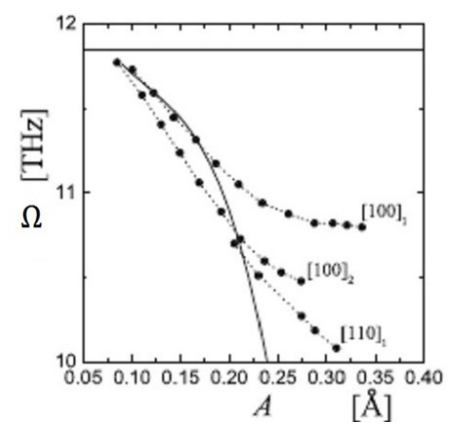

Fig. 6. Frequencies, as the functions of the DB amplitudes, A, for the DBs of three types: $[110]_{1},[100]_{1}$, and $[100]_{2}$, excited by simulations [19] for $m / M=0.1$, where figures in brackets describe polarization and the subscript indicates the number of the atoms oscillating with large amplitude. Solid curve gives $\Omega(A)$ found for the DB [100]1 in the frame of single degree of freedom model (see text). Horizontal line gives the upper edge of the phonon gap. Adapted from [19] Copyright by APS.

minimum equilibrium spacing between the light atoms along [110] is $4.4 \AA$, while the maximum DB [110] amplitude is about $0.31 \AA$. An attempt to increase its amplitudes beyond the maximal value leads to its transformation into the DB $[100]_{1}$, which becomes unstable upon further increase of the amplitude beyond $0.34 \AA$ and decays by radiating phonons [19].

High frequency DBs in $\mathrm{NaCl}$ type crystals (manifested by the peak above the phonon spectrum in Fig.3d) have not been modelled so far to our knowledge, but they have been found in a crystal of $\mathrm{Pt}_{3} \mathrm{Al}$ [36].

There are two main peculiarities of DBs related to the formation of coherent correlated states, namely, oscillations of atoms comprising a DB are (i) time-periodic and (ii) coherent, i.e. they have different amplitudes and commensurate frequencies. In particular, the central atom can vibrate with a large amplitude and frequency, while neighboring atoms vibrate with much smaller amplitudes and frequencies equal to the half of the main DB frequency [23]. Cosider a DB localized at one light atom that oscillates with a large amplitude, $\mathrm{A}$ in the anharmonic potential well, which determines its frequency $\Omega$ as follows [19]

$$
\begin{aligned}
& \Omega(A)=\sqrt{\alpha+\frac{3}{4} \beta A^{2}}, \alpha=\frac{2 \gamma_{1}}{m}, \beta=\frac{2 \gamma_{2}}{m} \\
& \gamma_{1}(A)=R_{1} A+S_{1}, \gamma_{2}(A)=R_{2} A+S_{2}
\end{aligned}
$$

where $\alpha$ determines the quasi-harmonic eigenfrequency of the potential well, and $\gamma_{1,2}$ describes its anharmonicity. Both of them depend on the DB amplitude, since it changes the force constants $\gamma_{1,2}$ of the potential. $\beta$ is positive, which corresponds to hard type of nonlinearity with frequency increasing with $\mathrm{A}$. However, the central atom oscillating with large amplitude shifts positions of neighboring atoms so that $\alpha$ decreases with A resulting in the observed decrease of $\Omega(A)$ shown in Fig.6. Solid curve shows $\Omega(A)$ for the DB [100] in this single degree of freedom model, which gives a good fit with 'exact' dashed curve for $\mathrm{A}<0.2 \AA$.

Let us apply this model to a DB in the PdD lattice, the DOS of which is shown in Fig.4. The lower optical phonon 


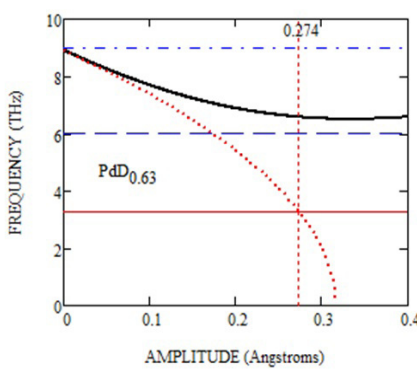

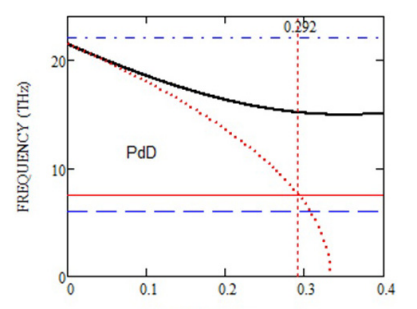

AMPLITUDE (Angstroms)

- DB frequency - Upper acoustic phonon edge Lower optic phonon edge Resonance frequency
Fig. 7. DB frequencies and eigenfrequencies of the potential wells as functions of the $\mathrm{DB}<110>$ amplitudes for different force constants assumed for $\mathrm{PdD}_{0.63}$ (a) and $\mathrm{PdD}$ (b) lattices. $\left.<110\right\rangle$ is the closepacked D-D direction.

frequency ( $\sim 9 \mathrm{THz})$ in Fig.4 is determined by the mass of $\mathrm{D}$, while the higher acoustic phonon frequency $(\sim 6 \mathrm{THz})$ is determined by the mass of $\mathrm{Pd}$ and by the corresponding force constants, which are different for D-D, D-Pd and Pd-Pd interactions and depend on $\mathrm{D}$ content.

Fig.7a shows $\mathrm{DB}<110>$ frequency $\Omega(A)$ given by Eq.(11) and eigenfrequency of (quasi-harmonic) potential wells for neighboring $\mathrm{D}$ atoms, $\omega_{0}(A)$ as functions of the $\mathrm{DB}$ amplitude:

$$
\omega_{0}(A)=\sqrt{\frac{2 \gamma_{1}(A)}{m}}, \gamma_{1}(A)=R_{1} A+S_{1}
$$

evaluated with the force constants assumed to fit DOS of $\operatorname{PdD}_{0.63}$ (Fig.4):

$$
\begin{aligned}
& \mathrm{R}_{1}=-0.026 \mathrm{eV} / \AA^{3}, \mathrm{~S}_{1}=0.008 \mathrm{eV} / \AA^{2}, \\
& \mathrm{R}_{2}=-0.017 \mathrm{eV} / \AA^{5}, \mathrm{~S}_{2}=0.035 \mathrm{eV} / \AA^{4}
\end{aligned}
$$

The frequency of the optic modes at the zone center $(\sim 9$ $\mathrm{THz}$ ), which determines the maximum DB frequency, is low in comparison with other hydrides, thus implying a weak nearest-neighbor Pd-D force constant in $\mathrm{PdD}_{0.63}$ [33]. One of the possible reasons for this broadness of the optic phonon spectrum is due to nonstoichiometry [37].

Fig.7b shows $\Omega(A)$ and $\omega_{0}(A)$ evaluated with the force constants fitted to raise the lower optic phonon edge for stoichiometric $\mathrm{PdD}$ up to $22 \mathrm{THz}$ and to broaden the phonon gap accordingly:

$$
\begin{aligned}
& \mathrm{R}_{1}=-0.143 \mathrm{eV} / \AA^{3}, \mathrm{~S}_{1}=0.048 \mathrm{eV} / \AA^{2}, \\
& \mathrm{R}_{2}=-0.013 \mathrm{eV} / \AA^{5}, \mathrm{~S}_{2}=0.143 \mathrm{eV} / \AA^{4}
\end{aligned}
$$

In the $\mathrm{PdD}_{0.63}$ case (Fig.7a), increase of the DB amplitude up to $0.274 \AA$ leads to the excitation in neighboring wells of the harmonic with the frequency $3.3 \mathrm{THz}$ equal to half of the main DB frequency $(\sim 6.6 \mathrm{THz})$, which interacts with acoustic phonons below the gap and makes the DB unstable. This case is similar to the $\mathrm{NaCl}$ type case for $\mathrm{m} / \mathrm{M}>0.2$ considered in [19].

In the PdD case (Fig.7b), increase of the DB amplitude up to the critical value $A_{\mathrm{cr}} \approx 0.292 \AA$ leads to the excitation in neighboring wells of the harmonic with the frequency $7.5 \mathrm{THz}$ equal to half of the main DB frequency $(\sim 15 \mathrm{THz})$, which lies above the upper acoustic phonon edge and does not interact with phonons. Such two-frequency DBs are stable, and they lead to the parametric formation of CCS of deuterons in the neighboring quasi-harmonic potential wells subjected to time-periodic modulation of their eigenfrequencies $\omega_{0}\left(A_{\mathrm{cr}}\right) \approx 7.5 \mathrm{THz}$ by the DB frequency $\Omega\left(A_{\mathrm{cr}}\right) \approx 15 \mathrm{THz}$. As a result of such modulation, $\mathrm{D}-\mathrm{D}$ fusion is expected to occur in several dozens of DB cycles (Fig.2) since the modulation amplitude $g_{\Omega} \approx A_{\mathrm{cr}} /\left(a_{\mathrm{PdD}}(2 / 2)^{0.5}\right) \approx 0.1$.

Thus, the $\mathrm{D}-\mathrm{D}$ fusion rate in $\mathrm{PdD}$ will be determined by the excitation rate of DBs having amplitudes near the critical value $A_{\mathrm{cr}}$, which will be evaluated in the following section.

\section{Excess power output under heavy water electrolysis}

The DB excitation occurs by thermal fluctuations and by external driving displacing atoms from equilibrium positions. The rate of thermal excitation of DBs having energy $E$ is given by Arrhenius law [27,31]

$$
K_{D B}^{t h}(E)=\omega_{D B} k_{D B}^{e f} \exp \left(-\frac{E}{k_{B} T}\right),
$$

where $k_{\mathrm{DB}}^{e f}$ is the efficiency coefficient for DB excitation, $k_{\mathrm{B}}$ is the Boltzmann constant, $T$ is the temperature, and $\omega_{\mathrm{DB}} \approx \Omega(0)$ is the attempt frequency that should be close to the edge of the phonon band, from which DBs are excited.

External driving of the DB excitation can be provided by focusing collisions and moving DBs (a.k.a. quodons) that are generated by knocking of surface atoms out of equilibrium position by energetic ions or molecules under electrolysis. The amplitude of the quasi-periodic energy deviation of atoms along the quodon pathway, $V_{\mathrm{ex}}$, can reach almost $1 \mathrm{eV}$ with the excitation time, $\tau_{\mathrm{ex}}$, of about 10 oscillation periods, which results in the amplification of $\mathrm{DB}$ generation rate proportional to the electric current density $J[31]$ :

$$
\begin{gathered}
K_{D B}^{J}(E)=K_{D B}^{t h}(E)\left(1+\left\langle I_{0}\left(\frac{V_{e x}}{k_{b} T}\right)\right\rangle \omega_{e x} \tau_{e x}\right) \\
\omega_{e x}\left(F_{q}\right)=F_{q} b^{2} \frac{3 l_{q}}{R_{P}}, F_{q}=\frac{J}{2 e}
\end{gathered}
$$

where $\omega_{e x}$ is the mean number of excitations per atom per second caused by the flux of quodons, $e$ is the electron charge, $b$ is the atomic spacing. The product $F_{q} b^{2}$ is the frequency of the excitations per atom within the layer of a thickness $l_{\mathrm{q}}$ equal to the quodon propagation range, while the ratio $3 l_{\mathrm{q}} / R_{\mathrm{p}}^{\mathrm{q}}$ is the geometrical factor that corresponds to the relative number of atoms within the quodon range in a PdD particle of a radius $R_{\mathrm{p}}$. The coefficient of proportionality between $F_{\mathrm{q}}$ and the electron flux $\mathrm{J} / \mathrm{e}$ assumes that each electrolytic reaction that involves a pair of electrons, releases a vibrational energy of $\sim 1 \mathrm{eV}$, which is sufficient for generation of one quodon with energy $V_{\text {ex }}<1 \mathrm{eV}$.

Multiplying the DB generation rate (18) by the tunneling 


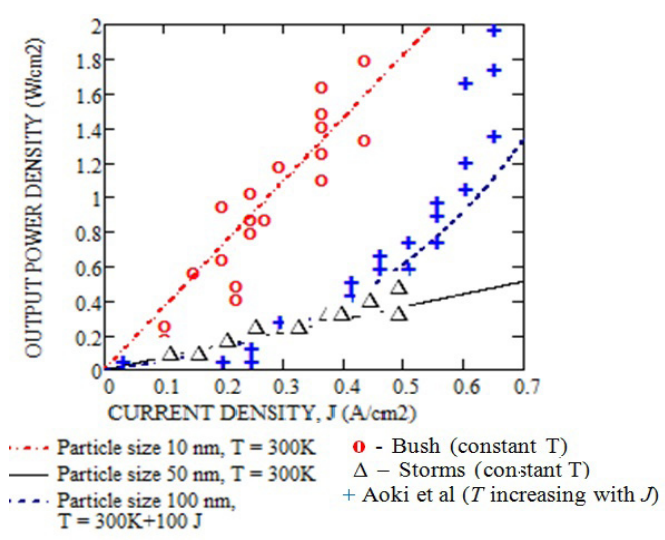

Fig. 8. LENR output power density according to the model Eq.(22) as a function of electric current density at constant $\mathrm{T}$ and $T$ increasing with $J$ as $T=300 \mathrm{~K}+100$ J. Experimental data [3], Fig.(42).

probability in a DB, $G^{*}(L, r)$ Eq.(10) and integrating over DB energies one obtains the $\mathrm{D}-\mathrm{D}$ fusion rate per PdD unit cell:

$$
R_{D D}=\frac{1}{\Delta E} \int_{E_{D B}^{*}-\Delta E}^{E_{D B}^{*}+\Delta E} K_{D B}^{J}(E) G^{*}(r) \mathrm{dE} \text {, }
$$

that dramatically depends on the correlation coefficient, $r$, which, in its turn, strongly depends on the DB amplitude $\mathrm{DB}$ energy and the number of DB cycles before decay, $n_{\mathrm{DB}}$.

Only a small fraction of DBs can form CCS in their vicinity and act as effective breather nano-colliders (BNC). They must have some particular energies, $E_{\mathrm{DB}}^{*} \pm \Delta E$, in order to cause the parametric resonance producing CCS. If $\Delta E<<k_{\mathrm{B}} T$, Eq.(19) is reduced to:

$$
\begin{aligned}
& R_{D D} \approx 2 K_{D B}^{J}\left(E_{D B}^{*}\right) G^{*}\left(r\left(E_{D B}^{*}, n_{D B}\right)\right) \\
& \underset{n_{D B}>n_{D B}^{*}}{\longrightarrow} K_{D B}^{J}\left(E_{D B}^{*}\right), G^{*}\left(r\left(E_{D B}^{*}, n_{D B}^{*}\right)\right) \approx \frac{1}{2}
\end{aligned}
$$

where the number of $\mathrm{DB}$ cycles required to make the Coulomb barrier 'transparent', $n_{\mathrm{DB}}^{*} \approx 100$ at $g_{\Omega}=0.1$.

Multiplying the DB-induced fusion rate (20) by the

Table 1. Material parameters used in the model

\begin{tabular}{|c|c|}
\hline Parameter & Value \\
\hline D-D equilibrium spacing in $\mathrm{PdD}, b(\AA)$ & 0.29 \\
\hline DB parametric amplitude, $A_{c \mathrm{cr}}(\AA)$ & 0.292 \\
\hline DB parametric energy, $E_{\mathrm{DB}}^{*}(\mathrm{eV})$ & 1 \\
\hline DB initial frequency, $\Omega(0)(\mathrm{THz})$ & 21 \\
\hline DB parametric frequency, $\Omega\left(A_{\mathrm{cr}}\right)(\mathrm{THz})$ & 15 \\
\hline DB-induced harmonic frequency, $\omega_{0}\left(A_{\mathrm{cr}}\right)(\mathrm{THz})$ & 7.5 \\
\hline Min. DB lifetime, $\tau_{e x}^{*}=n_{D B}^{*} / \omega_{D B}(\mathrm{~s})$ & $6 \times 10^{-12}$ \\
\hline DB excitation efficiency, $k_{D B}^{e f}$ & $4 \times 10^{-11}$ \\
\hline Quodon excitation energy $V_{e x}(\mathrm{eV})$ & 0.8 \\
\hline Quodon excitation time, $\tau_{e x}(\mathrm{~s})$ & $6 \times 10^{-13}$ \\
\hline Quodon propagation range, $l_{\mathrm{q}}=10 b(\mathrm{~nm})$ & 2.9 \\
\hline Cathode size/thickness $(\mathrm{mm})$ & 5 \\
\hline
\end{tabular}

energy $E_{\mathrm{D}-\mathrm{D}}=23.8 \mathrm{MeV}$, produced in $\mathrm{D}-\mathrm{D}$ fusion one obtains the excess energy production rate per atom, $P_{\mathrm{D}-\mathrm{D}}$ as a function of temperature and electric current:

$$
P_{D-D}(T, J)=K_{D B}^{J}\left(E_{D B}^{*}, T, J\right) E_{D-D},
$$

Usually, the output power density is measured per unite surface of a macroscopic cell, $P_{\mathrm{D}-\mathrm{D}}^{\mathrm{S}}$, as a function of the electric current density at a fixed temperature and at temperature increasing with $J$, as illustrated in Fig.8. This is given by the product of $P_{\mathrm{D}-\mathrm{D}}$, the number of atoms per unit volume, $1 / v_{\mathrm{PdD}}$

$\left(v_{\mathrm{PdD}}\right.$ being the atomic volume of $\left.\mathrm{PdD}\right)$ and the ratio of the cell volume to the cell surface:

$$
P_{D-D}^{S}(T, J)=P_{D-D}(T, J) \frac{L_{S}}{v_{P d D}},
$$

$L_{\mathrm{S}}$ is the cell size, if cubic, or thickness, in case of a plate.

Fig.8 shows the LENR output power density DBs as a function of electric current density and temperature evaluated by Eq.(22) assuming material parameters listed in Table 1. Comparison between the model and experimental data shows that the present model describes quantitatively the observed linear dependence of $P_{\text {D-D }}^{S}$ on the current density at a constant temperature as well as the deviation from the linear dependence, if temperature increases with increasing electric current density. Thermally-activated nature of the reactions leading to LENR has been noted for quite a long time [3], and the activation energy was estimated in some cases to be $\sim 0.65$ $\mathrm{eV}$. The present model not only explains these observations, but also reveals that the underlying physics is a consequence of the synergy between thermally activated and externally driven mechanisms of the $\mathrm{DB}$ excitation in deuterated palladium.

\section{Discussion}

The BNC concept proposed in a previous work [31] did not take into account correlation effects, and hence, unrealistically small separation between atoms $(\sim 0.01 \AA)$ would have to be attained in order to enhance the LENR rate up to a noticeable level. The main message of this paper is that DBs present the most efficient way to produce CCS due to time-periodic modulation of the potential well width (or the Coulomb barrier width) and hence to act as BNC triggering LENR in solids. Fig.9 demonstrates effect of CCS in the BNC model manifested by a number of DB cycles required to produce experimentally observed LENR rate $\sim 1 \mathrm{~W} / \mathrm{cm}^{2}$. It can be seen that in the modified model, the DB lifetime plays much more important role than the tunneling $\mathrm{D}-\mathrm{D}$ spacing, and that $\mathrm{DB}$ amplitude of several fractions of angstrom is sufficient to produce required effect, if CCS parametric conditions are met.

Medvedev et al [38] has demonstrated by means of MD simulations that gap DBs can be excited in the Al sub-lattice of $\mathrm{Pt}_{3} \mathrm{Al}$ under the action of time-periodic external driving. Time-periodic shaking of the surface atoms at frequencies near the optic phonon edge resulted in the DB excitation in the sub-surface layers. These findings point out at the possibility of LENR stimulation by external time-periodic excitation 


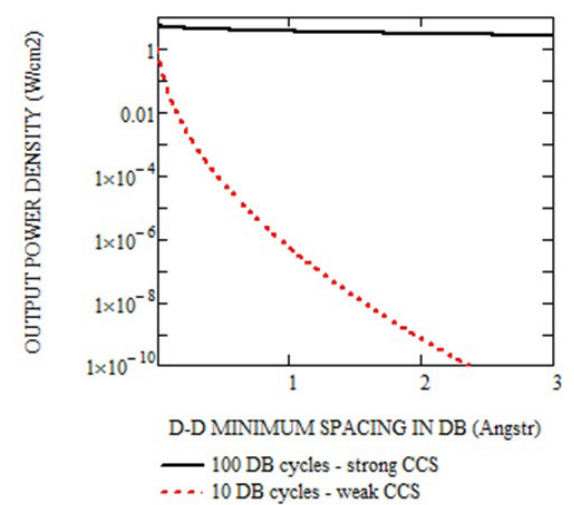

Fig. 9. LENR output power density according to Eq.(24) as a function of the tunneling D-D spacing at strong and weak CCS.

of surface atoms. That is what has been actually realized in «Terahertz» laser experiments [39] on the stimulation of nuclear reaction at a joint action of two low-power laser beams with variable beat frequency ranging from 3 to $24 \mathrm{THz}$ on the cathode surface during the $\mathrm{D}_{2} \mathrm{O}$ electrolysis in the $\mathrm{PdD}$ system.

Fig.10 shows the experimental frequency dependencies of the excess power in these experiments. Three main resonances of excess energy released at $\sim 8 \pm 1 \mathrm{THz}, 15 \pm 1 \mathrm{THz}$ and $21 \pm 1$ $\mathrm{THz}$ correlate with the DB-induced harmonic frequency, $\omega_{0}\left(A_{\mathrm{cr}}\right) \approx 7.5 \mathrm{THz}, \mathrm{DB}$ parametric frequency $\Omega\left(A_{\mathrm{cr}}\right) \approx 15 \mathrm{THz}$ and DB initial frequency, $21 \mathrm{THz}$, respectively (see the inset in the figure). According to the present model, the highest resonance is the biggest, since it is caused by amplification of $\mathrm{DB}$ excitation at the edge of optic phonon band. The medium resonance is due to tuning action of external driving on the DB frequencies: it increases the fraction of DBs with parametric frequency. The lowest resonance is due to tuning of harmonic frequencies by external driving: it increases the fraction of $\mathrm{D}$ atoms subjected to the parametric action by DBs.

The atoms are shaken by laser beams via excitation of the surface electron plasmons as suggested in [39]. It explains the necessity of external magnetic field for producing

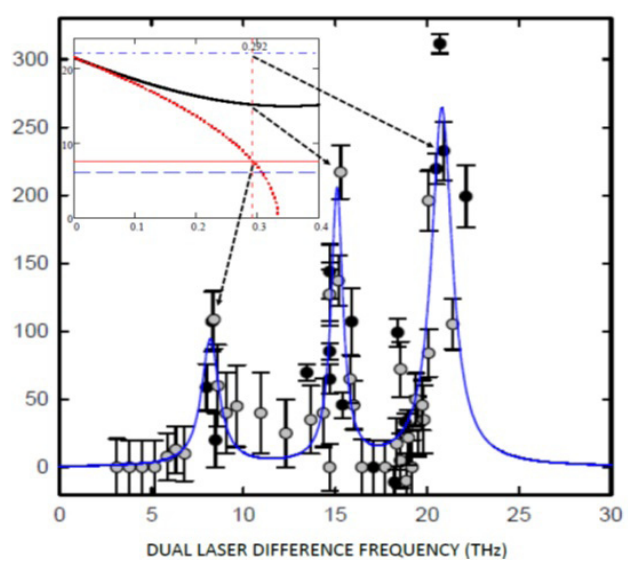

Fig. 10. Excess power (mW) under joint action of two low-power laser beams with variable beat frequency on the surface of the Pd cathode during the electrolysis in heavy water [39]. The inset (from Fig. 7b) shows parity between critical DB-induced frequencies and the resonance frequencies [39] designated by dashed arrows. resonance effects $[39,13]$. However, the direct modulation of the frequency of the optical phonon modes by plasmons proposed in [13] as a driving force for the CCS formation is very questionable [31], and it does not explain LENR observed in the absence of the laser driving at slightly higher electric current or temperature [39]. In the present model, the laser driving acts just as a tuning tool for the CCS formation by $\mathrm{DBs}$ induced by temperature and electric current.

One of the new and important consequences of the present model is that it offers a principal explanation for the critical D loading required for LENR. It is known that excess heat usually does not appear until the loading is near 0.83 [39], whereas neuron scattering measurements of the phonon spectra have been done for non-stoichiometric $\mathrm{PdD}_{0.63}$, the DOS of which (Fig.7a) does not support formation of CCS by the present mechanism, since its phonon gap is not sufficiently broad. Our hypothesis is that mechanical stresses arising in $\mathrm{PdDx}$ above the critical loading $\mathrm{x}>0.83$ can make the phonon band similar to that shown in Fig.7b thus switching on the DB-induced formation of CCS.

Structural information on ball milled magnesium hydride from vibrational spectroscopy and ab-initio calculations [40] has shown that the high-energy part of the vibrational spectrum is rather sensitive to stresses induced by for instance ball milling. The structure of PdD at extreme loading is similar to that after the ball milling, which seems to support the present hypothesis.

Another factor concerning the role of the crystal disorder in LENR is a striking site selectiveness of DB formation in the presence of spatial disorder $[26,30,41]$. It means that the process of loading or special 'nano-treatment' creates the disordered cluster structures, which may be enriched with sites of zero or small threshold energies for the DB excitation. Such sites are expected to become the nuclear active cites, according to the present model.

\section{Conclusions and outlook}

Persistent spatially localized vibrations of nonlinear origin known as discrete breathers (DBs) that can be excited generically in many-body nonlinear systems are proposed to produce coherent correlation states (CCS) due to timeperiodic modulation of the potential well width and hence to act as breather 'nano-colliders' (BNC) triggering low energy nuclear reactions in solids. In particular, tunneling probability for deuterium (D-D) fusion in 'gap DBs' formed in metal deuterides has been shown to increase with increasing number of oscillations by $\sim 190$ orders of magnitude resulting in the observed LENR rate at extremely low concentrations of DBs.

The present model explains all the salient LENR requirements: (i) high loading of $\mathrm{D}$ within the Pd lattice as preconditioning needed to prepare $\mathrm{PdD}$ crystallites with appropriate DOS, and (ii) the triggering by D flux or electric current, which facilitates the DB creation by the input energy transformed into the lattice vibrations.

The present model describes the observed linear dependence of the excess power output on the current density under heavy water electrolysis at a constant temperature as 
well as its exponential increase with increasing temperature, which can be the basic LENR mechanism in the hot CATtype installations.

The proposed mechanism of CCS formation near the gap DBs requires sufficiently broad phonon gap that is not observed below the critical D loading $\sim 0.83$ examined so far. Further investigations of DOS and DBs in the extreme conditions of LENR are required.

The present results are based only on the known physical principles and on independent atomistic simulations of DBs in metals and ion crystals using realistic many-body interatomic potentials. Atomistic modeling of DBs of various types in metal hydrides/deuterides is an important outstanding problem since it may offer the ways of engineering the nuclear active environment.

Acknowledgements. The author is grateful to Sergey Dmitriev and Vladimir Vysotskii for helpful discussions and valuable criticism.

\section{References}

1. M. Fleischmann, S. Pons, M. Hawkins, J. Electroanal. Chem. 261, 301-308 (1989).

2. M. McKubre, F. Tanzella, P. Hagelstein, K. Mullican, M. Trevithick. In: Tenth International Conference on Cold Fusion. MA: LENR-CANR.org.. Cambridge (2003) $1-13 \mathrm{p}$.

3. E. K. Storms. The science of low energy nuclear reaction, World Scientific. Singapore (2007) 312 p.

4. A. G.Parkhomov. International Journal of Unconventional Science. 7 (3), 68-72 (2015).

5. H. J. Assenbaum, K. Langanke C. Rolfs. Z. Phys. A 327, 461-468 (1987).

6. J. Kasagi. Screening In: ICCF-14 International Conference on Condensed Matter Nuclear Science (2008).

7. E. Schrödinger. Ber. Kgl. Akad. Wiss. Berlin (1930) 296$303 \mathrm{p}$.

8. H. P. Robertson. Phys. Rev. 34, 163-164 (1930).

9. V. V. Dodonov, V. I. Man'ko. Phys. Lett. A 79 (2/3), 150152 (1980).

10. V.I. Vysotskii, S. V. Adamenko. J. Tech. Phys. 55, 613621 (2010).

11. V.I. Vysotskii, M.V. Vysotskyy, S.V. Adamenko. J. Exp. Theor. Phys. 141, 276-287 (2012).

12. V.I. Vysotskii, S.V. Adamenko, M.V. Vysotskyy. J. Exp. Theor. Phys. 142, 627-643 (2012).

13. V.I. Vysotskii, M. V. Vysotskyy. Eur. Phys. J. A (2013). DOI 10.1140/epja/i2013-13099-2

14. A. J. Sievers and S. Takeno, Phys. Rev. Lett. 61, 970-973 (1988).

15. S. Flach, A. V. Gorbach, Phys. Rep. 467, 1-116, (2008).

16. V. Hizhnyakov, D. Nevedrov, A. J. Sievers. Physica. B. 316-317, 132-135 (2002).

17. M.E. Manley,A. J. Sievers, J.W. Lynn, S.A. Kiselev,
N. I. Agladze, Y. Chen, A. Llobet, A. Alatas. Phys. Rev. B 79, 134304-1-5 (2009).

18. M. E. Manley. Acta Materialia. 58, 2926-2935 (2010).

19. L. Z. Khadeeva, S. V. Dmitriev. Phys. Rev. B 81, 214306-1-8 (2010).

20. AA. Kistanov, S. V. Dmitriev. Phys. Solid State. 54, 16481651 (2012).

21. L. Z. Khadeeva, S. V. Dmitriev. Phys. Rev. B 84, 144304-1-8 (2011).

22. S. V. Dmitriev, A. P Chetverikov, M. G Velarde. Physica status solidi. (b), $1-5$ (2015). DOI: 10.1002/pssb.201451673

23. G. M. Chechin, G. S. Dzhelauhova. J. Sound and Vibration. 322, 490-512 (2009).

24. M. Haas, V. Hizhnyakov, A. Shelkan, M. Klopov, A. J. Sievers. Phys. Rev. B 84, 14430-1-8 (2011).

25. D. Terentyev, A. Dubinko, V. Dubinko, S. Dmitriev, E. Zhurkin, M. Sorokin. Interaction of discrete breathers with primary lattice defects in $b c c$ Fe. Modelling Simul. Mater. Sci. Eng., to be published.

26. F. Piazza, Y.H. Sanejouand. Phys. Biol. 5, 026001-1-14 (2008).

27. V.I. Dubinko, P.A. Selyshchev, and J.F. R. Archilla, Phys. Rev. E 83 (4) 124-126 (2011). DOI: 10.1103/PhysRevE.83.041124

28. V.I. Dubinko, F. M. Russell. J. Nuclear Materials. 419, 378-385 (2011).

29. V.I. Dubinko, A. V. Dubinko. Nuclear Inst. and Methods in Physics Research. B 303, 133-135 (2013).

30. V. I. Dubinko, F. Piazza. Letters on Materials. 4 (4), $273-$ 278 (2014).

31. V. I. Dubinko. J. Condensed Matter Nucl. Sci. 14, 87-107 (2014).

32. P. Hanggi, P. Talkner, M. Borkovec. Rev. Mod. Phys. 62, 251-341 (1990).

33. J.M. Rowe, J. J. Rush, H.G. Smith, M. Mostoller, H. E. Flotow. Phys. Rev. Lett. 33, 1297-1300 (1974).

34. I. Errea, M. Calandra1, F. Mauri, Phys. Rev. Lett. 111, 177002-1-5 (2013).

35. S. Kanagaprabha, A. Meenaatci, R. Rajeswarapalanichamy, K. Iyakutti. WJST. 9 (2), 1-12 (2012).

36. P.V. Zakharov, M.D. Starostenkov, S.V. Dmitriev, N. N. Medvedev, A. M. Eryomin. Modeling the interaction of discrete breathers of various types in nanowires of crystal $\mathrm{Pt}_{3} \mathrm{Al}$. JETP, to be published.

37. A. Rahman, K. SkoId, G. Pelizarri, S. K. Sinha, H. Flotow. Phys. Rev. B 14, 3630-3634 (1976).

38. N.N. Medvedev, M.D. Starostenkov, P.V. Zakharov, O. V. Pozhydaeva. J. Tech. Phys. Letters. 37, 7-15 (2011).

39. P. L. Hagelstein, D. Letts, D. Cravens. J. Condensed Matter Nucl. Sci. 3, 59-76 (2010).

40. H.G. Schimmel, M.R. Johnson, G. J. Kearley, A. J. Ramirez-Cuesta, J. Huot, F. M. Mulder, J. Alloys and Compounds. 393, 1-4 (2005).

41. H. Zhang, J. F. Douglas. Soft Matter. 9, 1266-1280 (2013). 